\title{
Kidney Injury Molecule-1 and Periostin Urinary Excretion and Tissue Expression Levels and Association with Glomerular Disease Outcomes
}

\author{
Qiaoyan Wu ${ }^{a, b}$ Jonathan P. Troost ${ }^{c}$ Tiane Dai ${ }^{a} \quad$ Cynthia Nast $^{d}$ \\ Sean Eddy ${ }^{e}$ Boxian Wei ${ }^{f}$ Ying Wang ${ }^{a}$ Debbie S. Gipson ${ }^{f}$ Katherine M. Dellg \\ Keisha L. Gibson ${ }^{\mathrm{h}}$ Matthias Kretzler ${ }^{\mathrm{e}}$ Sharon Adler ${ }^{\mathrm{a}}$ \\ aDivision of Nephrology and Hypertension, Los Angeles Biomedical Research Institute at Harbor-University of \\ California, Torrance, CA, USA; ${ }^{b}$ Department of Nephrology, The First Affiliated Hospital, Guangxi Medical University, \\ Nanning, China; 'Michigan Institute for Clinical and Health Research, University of Michigan, Ann Arbor, MI, USA; \\ ${ }^{d}$ Cedars-Sinai Medical Center, Los Angeles, CA, USA; 'Division of Nephrology, Department of Internal Medicine, \\ University of Michigan, Ann Arbor, MI, USA; fDivision of Nephrology, Department of Pediatrics, University of \\ Michigan, Ann Arbor, MI, USA; ${ }^{9}$ Department of Pediatrics, Case Western Reserve University and Cleveland Clinic \\ Children's, Cleveland, OH, USA; ' University of North Carolina Kidney Center, Chapel Hill, NC, USA
}

\section{Keywords}

Kidney injury molecule-1 · Periostin · Proteinuria $\cdot$ Renal morphology · Glomerular disease

\begin{abstract}
Introductions: Kidney injury molecule-1 (KIM-1) and periostin (POSTN) are proximal and distal tubule injury biomarkers. We tested whether baseline urine $\mathrm{KIM}-1 / \mathrm{Cr}(\mathrm{UKIM}-1 / \mathrm{Cr})$ and/ or UPOSTN/Cr correlated with disease severity or improved a remission prediction model. Methods: Baseline uKIM-1/Cr and UPOSTN/Cr were measured on spot urine samples from immunosuppression-free patients enrolled in Nephrotic Syndrome Study Network until December 15, 2014. Urine protein/ $\mathrm{Cr}$ (UPCR) and albumin/Cr (UACR) were measured at baseline, 4 months, and until last follow-up. Glomerular and tubulointerstitial (TI) expression arrays were analyzed from a baseline research renal biopsy core collected during a clinically indicated biopsy. Renal diagnoses were centrally confirmed, sections scanned, and measured morphometrically. Correlations between baseline uKIM-1/Cr and uPOSTN/Cr
\end{abstract}

karger@karger.com www.karger.com/gdz

Karger $\stackrel{\text { ' }}{5}$

BOPEN ACCESS
(C) 2021 The Author(s)

Published by S. Karger AG, Basel

This article is licensed under the Creative Commons AttributionNonCommercial-NoDerivatives 4.0 International License (CC BY NC-ND) (http://www.karger.com/Services/OpenAccessLicense) Usage and distribution for commercial purposes as well as any distribution of modified material requires written permission. and UPCR, UACR, histopathologic features, glomerular and TI KIM-1 and POSTN expression levels, and renal outcomes were assessed. Results: Baseline uKIM-1/cr correlated with UPCR and UACR and were associated with complete remission (CR) after adjustment for proteinuria, histopathologic diagnosis, and treatment. Baseline uKIM-1/Cr also correlated with degree of foot process effacement and acute tubular injury. Glomerular and TI KIM-1 expression levels correlated with UPCR and UACR. Higher TI KIM-1 expression levels correlated with interstitial fibrosis, tubular atrophy, and global glomerulosclerosis, while glomerular KIM-1 expression correlated with time to remission. Findings for POSTN were of lesser statistical strength. Discussion/Conclusion: Lower baseline UKIM-1/Cr values were associated with more rapid time to $C R$ after adjusting for proteinuria, histopathologic diagnosis, and treatment. Increased TI KIM-1 expression levels in proteinuric states were associated with chronic morphological injury; lower glomerular expression levels were associated with a greater potential for proteinuria reversibility.

(c) 2021 The Author(s)

Published by S. Karger AG, Basel
Jonathan P. Troost

Michigan Institute for Clinical \& Health Research, University of Michigan 2800 Plymouth Road, Bldg 400

Ann Arbor, MI 48109-2800 (USA)

troostj@med.umich.edu 


\section{Introduction}

Tissue and urine kidney injury molecule-1 (KIM-1) and periostin (POSTN) have been implicated as markers of acute kidney injury (AKI) and CKD [1-4]. In the kidney, KIM-1 is expressed predominantly in proximal renal tubules and mediates autophagy in response to injury [57]. POSTN is expressed predominantly in distal tubules and is a marker and mediator of tubular dedifferentiation [3]. While these markers may identify AKI earlier than serum $\mathrm{Cr}$ [8-10], the value of novel tissue and/or urine markers that correlate with and/or predict outcomes for CKD has generally been disappointing $[11,12]$. Measures that add value to clinical predictors of treatment response and prognosis in patients with glomerular disease are needed. Chronic tubulointerstitial (TI) disease as a consequence of proteinuria is a key factor determining progressive loss of renal function. Thus, measures of tubular injury might associate with and/or predict clinical outcomes, as well as provide insights into the mechanisms of progressive renal injury.

The Nephrotic Syndrome Study Network (NEPTUNE) is a multicenter consortium utilizing a systems biology approach to elucidate the pathogenesis of minimal change disease (MCD), focal and segmental glomerulosclerosis (FSGS), and membranous nephropathy $(\mathrm{MN})$ by incorporating clinical features, morphologic change, expression arrays, metabolomics, proteomics, and genomics to develop novel molecular signatures for these diseases [13]. Ideally, targeted treatment strategies are anticipated to be derived from these molecular signatures.

\section{Materials and Methods}

\section{Subjects}

NEPTUNE recruited a cohort of pediatric and adult subjects enriched for FSGS, MCD, and MN. Due to expected imprecision of pre-biopsy clinical diagnoses, patients with other diagnoses were also collected and classified as "other glomerulopathies." A full descriptor of subject inclusion and exclusion criteria are published [13]. All NEPTUNE subjects were enrolled at the time of clinically indicated renal biopsies. For the purposes of the current study, urine assays were performed only on subjects who were not taking steroids or other immunosuppressive medications anytime within a 30-day window prior to the baseline biopsy $(n=50)$. To enhance power, TI and/or glomerular expression data for KIM-1 and/or POSTN were tested for correlations with morphologic data in all NEPTUNE I patients who were not taking immunosuppressive medications at the time of the baseline urine expression and for whom expression data were available $(n=181)$. This research was carried out according to the Code of Ethics of the World Med- ical Association (Declaration of Helsinki), and study protocol was reviewed and approved by the University of Michigan Institutional Review Board (HUM00158219). Samples obtained for this study were de-identified and obtained from the central NEPTUNE repository.

\section{Urine Collection and Storage}

For measurements of urine KIM-1 and POSTN, urine aliquots from spot urine samples were obtained at the baseline visit. For urine albumin/ $\mathrm{Cr}$ and urine protein/Cr, samples were obtained at baseline and at 4-6 month intervals until the last follow-up samples available were assayed. All urine samples were collected, processed, stored, and shipped according to a standard NEPTUNE urine collection protocol [14]. Briefly, $12 \mathrm{~mL}$ of clean catch urine was mixed with $15 \mu \mathrm{L}$ of protease inhibitor cocktail (Sigma P1860, which includes as active agents aprotinin, bestatin, E-64, leupeptin, and pepstatin A), centrifuged, and supernatant was aliquoted, for a final concentration of $1.25 \mu \mathrm{L} / \mathrm{mL}$. Urines were stored at $-80^{\circ} \mathrm{C}$, shipped for frozen analysis.

\section{Urine KIM-1 and POSTN Assays}

Human urine KIM-1 was measured by a commercial ELISA (Enzo Life Sciences, Inc. Farmingdale, NY, USA). The measurement was performed according to the manufacturer's protocol using undiluted urine and also urine diluted from 1:5 to 1:10 in assay buffer. Samples were analyzed spectrophotometrically at $450 \mathrm{~nm}$ of absorbance. For the urine POSTN measurements, a separate urine purification step was performed to ensure that the POSTN kit standards and the urine sample spectrophotometric measurements were similar in background. For the purification step, 250 $\mu \mathrm{L}$ of urine was applied to Microcon 10 ultrafiltration membranes, then centrifuged at $14,000 \mathrm{rpm}$ at $4^{\circ} \mathrm{C}$ for $30 \mathrm{~min}$. Concentrated urine was washed with $10 \mathrm{mM}$ Tris- $\mathrm{HCl}(\mathrm{pH} \mathrm{8.0)}$, ultrafiltered twice to remove residual small molecules including salts, and purified samples were diluted in reagent to adjust the final volume to $250 \mu \mathrm{L}$. A human POSTN DuoSet ELISA Development System kit (R\&D Systems, Minneapolis, MN, USA) was used according to the manufacturer's instructions. Samples were analyzed spectrophotometrically at $450 \mathrm{~nm}$ of absorbance. Results were expressed normalized to locally measured urine $\mathrm{Cr}$ measured in duplicate.

Urine Protein and Albumin Assays

Urine protein:Cr ratio (UPCR) and urine albumin:Cr ratio (UACR) values were centrally measured; 43 of 50 were from timed urines, and 7 were from spot urine. Of the 265 follow-up measurements used in these analyses, 89\% were centrally measured: 197 from timed and 39 from spot urines; the remaining 29 samples were locally measured and reported. Categorical designations of complete remission (CR), partial remission (PR), and no remission (NR) were assigned by the NEPTUNE Data Coordinating Center. CR was defined as a UPCR $<0.3 \mathrm{~g} / \mathrm{g}$ and PR as a $50 \%$ reduction in UPCR from visit 1 and UPCR $<3.5 \mathrm{~g} / \mathrm{g}$. Patients not meeting the CR or PR definitions were defined as NR.

Fig. 1. Flow diagram of included patients. NEPTUNE-biopsied patients enrolled between July 2010 and August 2015*. NEPTUNE, Nephrotic Syndrome Study Network; KIM-1, kidney injury molecule; POSTN, periostin.
(For figure see next page.)
Glomerular Dis 2021;1:45-59 DOI: $10.1159 / 000513166$
Wu/Troost/Dai/Nast/Eddy/Wei/Wang/ Gipson/Dell/Gibson/Kretzler/Adler 
Urine sample

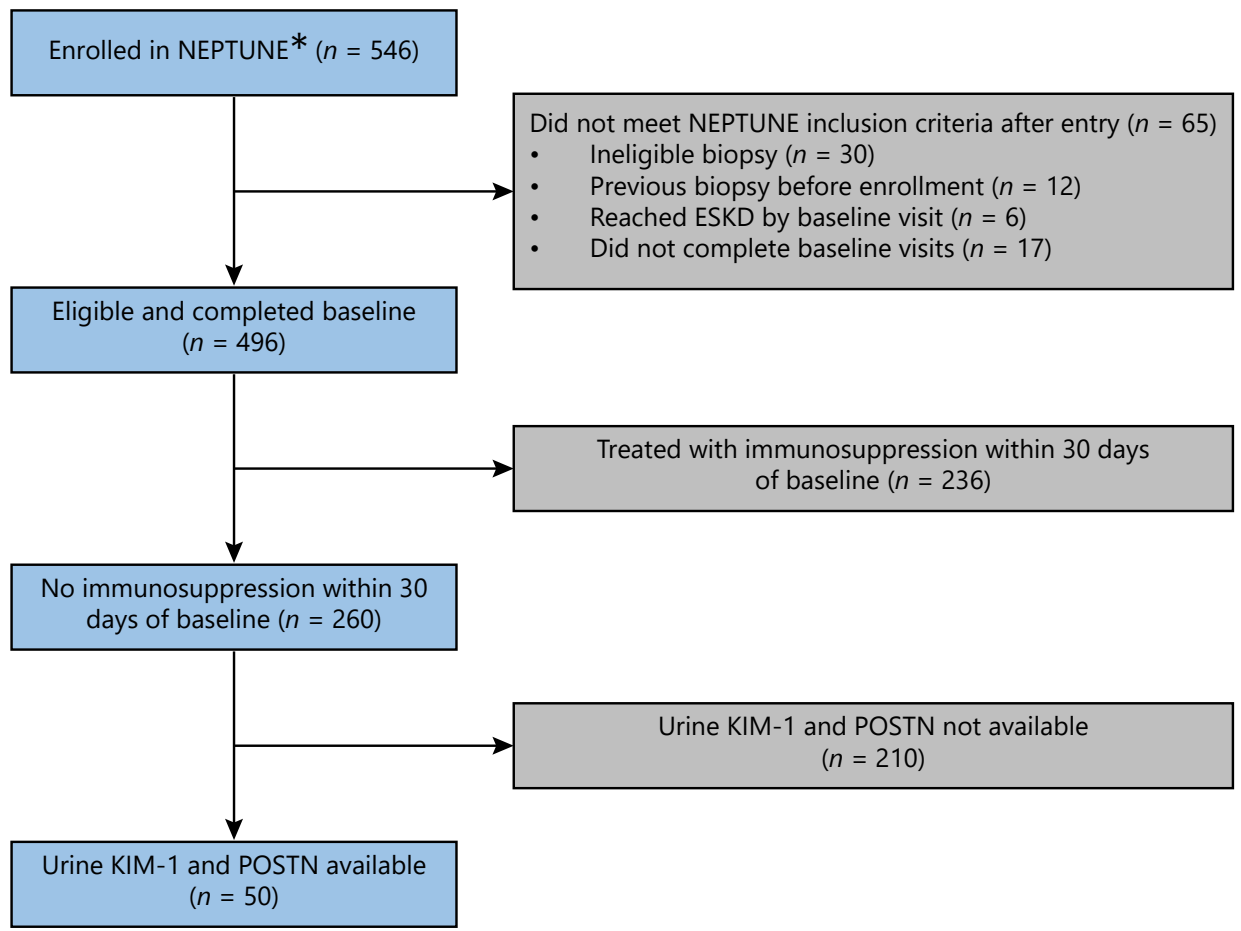

a *Urine cutoff 12/15/2014

\section{Expression sample}

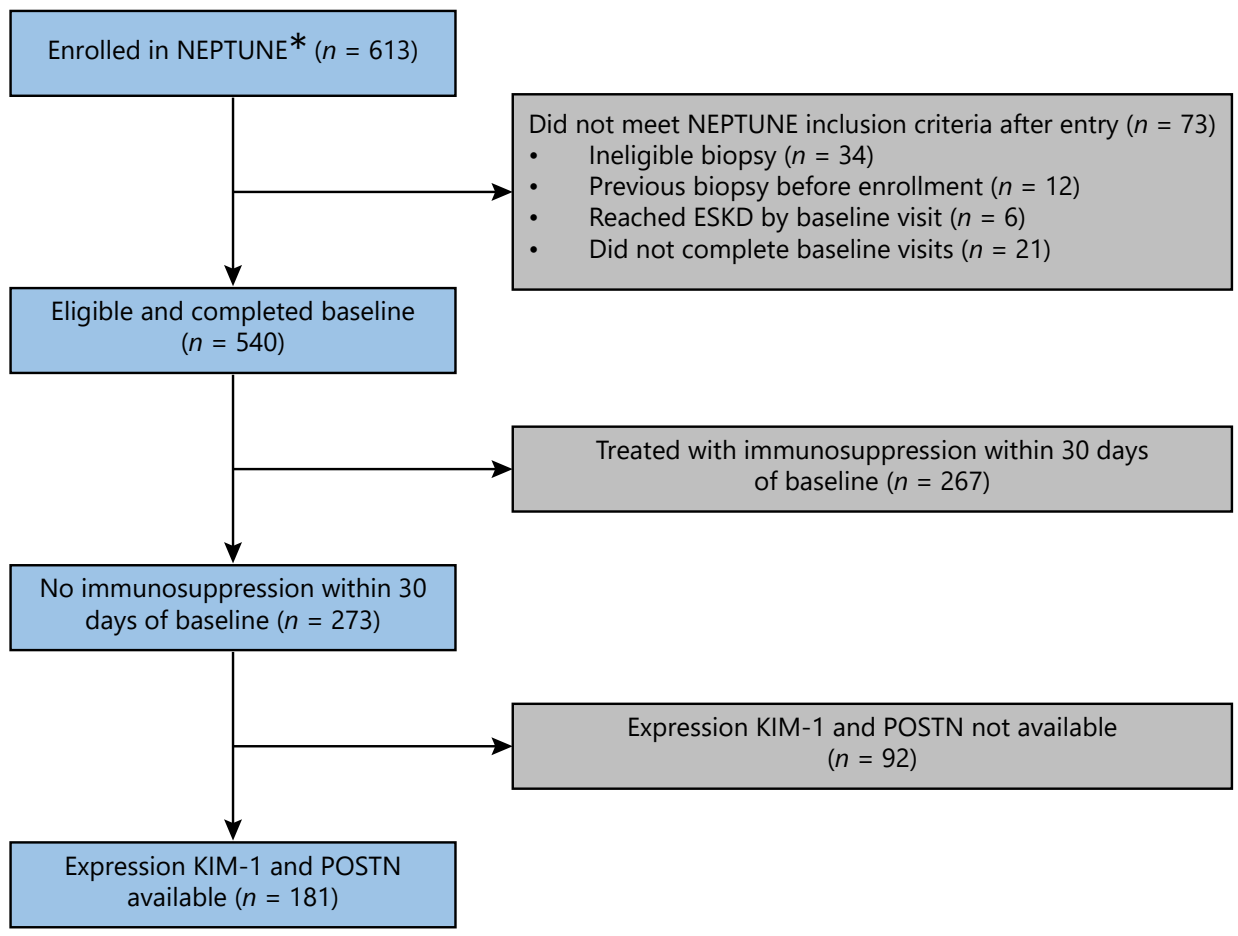

b *xpression cutoff 02/11/2016 


\section{TI KIM-1 and POSTN Expression}

A single renal biopsy core was immediately placed in RNAlater (Qiagen) and frozen at $-80^{\circ} \mathrm{C}$ until shipped to the central site (University of Michigan, Ann Arbor). Samples were then thawed, and glomeruli were microdissected and separated from tubulointerstitium, using defined protocols. Total RNA was isolated, reverse transcribed, linearly amplified, and hybridized on an Affymetrix 2.1 ST platform [15-17]. Gene expression was normalized, quantified, and annotated at the Entrez gene level.

\section{Renal Morphology Evaluation}

All subjects' diagnoses were reviewed and confirmed for accuracy and consistency by NEPTUNE renal pathologists. Morphology was annotated by NEPTUNE pathologists on digitally scanned slides according to the whole slide imaging techniques previously described [18]. Urine assay results were correlated with quantitative morphological findings.
Statistical Analyses

Descriptive analyses were performed using frequencies and percentages for categorical variables and median and intraquartile ranges for continuous variables. The descriptive characteristics of the urine and expression samples were compared using $\chi^{2}$ and KruskalWallis tests. Natural $\log$ transformations $\left(\log _{2}\right)$ were used when displaying urine biomarkers (urine $\mathrm{KIM}-1 / \mathrm{Cr}$ [uKIM-1/Cr] and urine POSTN/Cr), UPCR, and UACR to normalize skewed distributions. Glomerular and tubular transcript variables are calculated on a log-2 scale. Unadjusted associations between urine biomarkers and diagnosis, proteinuria, estimated glomerular filtration rate (eGFR), sclerosis, segmental sclerosis, interstitial fibrosis, and tubular atrophy, and foot process effacement using scatter plots and Spearman correlation coefficients for continuous comparisons; boxplots and Kruskal-Wallis tests were used for categorical comparisons. Cox proportional hazards models were used to assess if urine biomarkers correlated with time to first CR. Models were performed by treating

Table 1. Descriptive characteristics of NEPTUNE participants: comparison of urine and expression samples

\begin{tabular}{|c|c|c|c|}
\hline Characteristic & $\begin{array}{l}\text { Urine sample } \\
(n=50)\end{array}$ & $\begin{array}{l}\text { Expression sample } \\
(n=181)\end{array}$ & $p$ value \\
\hline Age at baseline, median (IQR) & $42(26,49)$ & $43(27,58)$ & \\
\hline Children (age < 18$), n(\%)$ & $7(14)$ & $25(14)$ & 0.36 \\
\hline Female, $n(\%)$ & $23(46)$ & $63(35)$ & 0.15 \\
\hline \multicolumn{4}{|l|}{ Race, $n(\%)$} \\
\hline Asian/Asian American & $7(14)$ & $21(12)$ & \multirow{4}{*}{0.93} \\
\hline Black/African American & $13(26)$ & $46(25)$ & \\
\hline White/Caucasian & $26(52)$ & $102(56)$ & \\
\hline Other & $4(8)$ & $12(7)$ & \\
\hline \multicolumn{4}{|l|}{ Diagnosis, $n(\%)$} \\
\hline MCD & $8(16)$ & $25(14)$ & \multirow{4}{*}{0.51} \\
\hline FSGS & $21(42)$ & $59(33)$ & \\
\hline $\mathrm{MN}$ & $11(22)$ & $46(25)$ & \\
\hline Other glomerulopathy & $10(20)$ & $51(28)$ & \\
\hline Urine protein:Cr ratio at eligibility, g/g, median (IQR) & $2.7(1.2,6.2)$ & $3.2(1.7,6.2)$ & \\
\hline$>3.0 \mathrm{~g} / \mathrm{g}, n(\%)$ & $24(48)$ & $98(54)$ & 0.43 \\
\hline eGFR at baseline, $\mathrm{mL} / \mathrm{min} / 1.73 \mathrm{~m}^{2}$, median (IQR) & $75(34,102)$ & $75(49,101)$ & 0.59 \\
\hline \multicolumn{4}{|l|}{ Treatment after baseline, $n(\%)$} \\
\hline Steroids & $14(28)$ & $75(41)$ & 0.08 \\
\hline Calcineurin inhibitors & $5(10)$ & $23(13)$ & 0.60 \\
\hline Mycophenolate mofetil & $7(14)$ & $17(9)$ & 0.34 \\
\hline Cyclophosphamide & $1(2)$ & $11(6)$ & 0.25 \\
\hline Total months of follow-up, median (IQR) & $34(14,54)$ & $43(23,55)$ & 0.19 \\
\hline
\end{tabular}

eGFR, estimated glomerular filtration rate; IQR, interquartile range; NEPTUNE, Nephrotic Syndrome Study Network; MCD, minimal change disease; MN, membranous nephropathy; FSGS, focal segmental glomerulosclerosis.

Fig. 2. Baseline urine KIM-1 correlated with proteinuria, albuminuria, kidney diagnosis, and foot process effacement and was predictive of time to CR. a KIM- 1 *UPCR (Spearman's rho $=0.75 ; p<$ $0.001)$. b KIM- $1 *$ UACR (Spearman's rho $=0.74 ; p=0.002)$. $\mathbf{c}$ KIM$1(p$ overall $=0.01 ; p$ MCD vs. all others $=0.04)$. d KIM- $1 *$ foot process effacement (Spearman's rho $=0.59 ; p<0.001$ ). e KIM-
$1^{*}$ acute tubular injury (Spearman's rho $=0.31 ; p=0.04$ ). f KIM$1 *$ time to complete remission $(p=0.01)$. FSGS, focal segmental glomerulosclerosis; KIM-1, kidney injury molecule; CR, complete remission; $\mathrm{MCD}$, minimal change disease; $\mathrm{MN}$, membranous nephropathy; UACR, urine albumin:Cr ratio; UPCR, urine protein:Cr ratio; uKIM-1/UCR, urine KIM-1/urine Cr concentration.

(For figure see next page.)
48

Glomerular Dis 2021;1:45-59

DOI: $10.1159 / 000513166$
Wu/Troost/Dai/Nast/Eddy/Wei/Wang/

Gipson/Dell/Gibson/Kretzler/Adler 


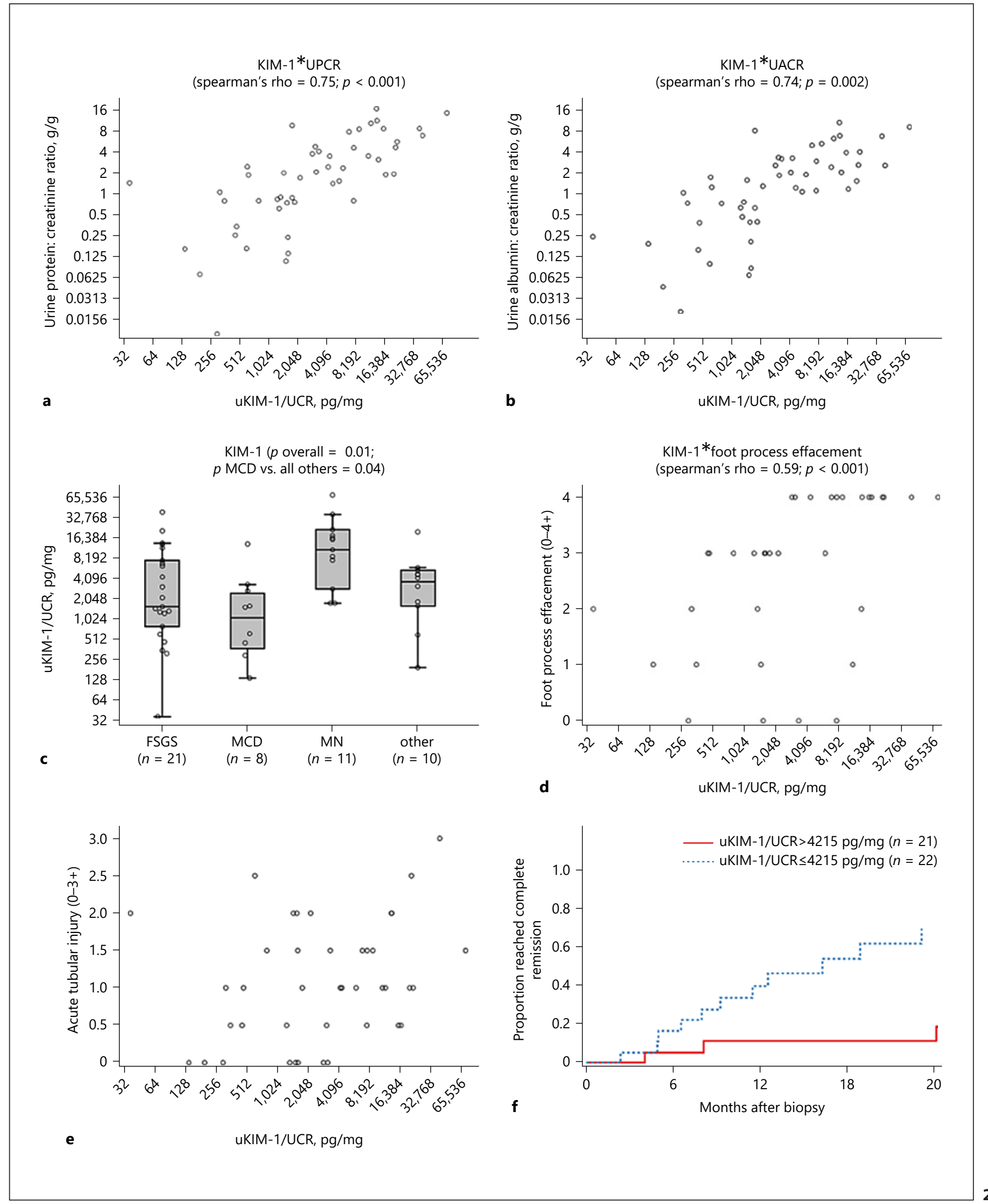


each biomarker as a continuous variable on a log-2 scale and also separately by dichotomizing as a median split. Analyses were adjusted for cohort, UPCR, and steroid therapy. Full-adjusted models were reported as well as reduced model after backward selection. To assess the improvement in prediction associated with biomarker variables, -2 log likelihood ratio tests were performed to compare multivariable models to the same multivariable model after dropping the biomarker variable of interest. Harrell's concordance indices, a marker of goodness of fit for survival models analogous to a C-statistic or "area under the curve" from a logistic regression, were also reported. Analyses were performed in SAS v9.4.

\section{Results}

\section{Participant Characteristics}

Figure 1 is a flow diagram of the included participants. Of the 546 biopsied patients enrolled between July 2010 and December 2014, 65 participants did not meet inclusion criteria after entry: 30 had ineligible biopsy diagnoses (9 diabetic nephropathy, 9 nondiagnostic biopsy, 4 vasculitis, 4 immune complex mediated glomerulonephritis, 3 amyloidosis, and 1 abnormalities of glomerular basement membrane); 12 had previous biopsies; 6 had reached ESRD at the time of enrollment; and 17 withdrew before completing all screening, baseline, and biopsy visits. Of the remaining 496, 236 were exposed to immunosuppressive therapy in the 30 days before baseline and were not eligible for these analyses. Of the remaining 260, urine biomarkers were measured on urine from the 50 participants available at the start time of this study. mRNA expression data were gathered among those enrolled by February 2016 and were available on 181 with tissue microdissections.

Baseline characteristics of these samples are shown in Table 1. The urine sample included $8 \mathrm{MCD}, 21$ FSGS, 11 $\mathrm{MN}$, and 10 other glomerulopathies. The sample was $52 \%$ White/Caucasian and had a median age of 42 , median UPCR 2.7, and median eGFR of $75 \mathrm{~mL} / \mathrm{min} / \mathrm{m}^{2}$. There were no significant differences in the baseline characteristics of subjects contributing to the urine and expression samples.

\section{Urine KIM-1}

By univariate analysis, uKIM-1/Cr correlated with UPCR (Fig. 2a, rho $=0.75, p<0.001$ ) and UACR (Fig. 2b, rho $=0.74, p=0.002$ ) but not with baseline eGFR (see online suppl. Fig. 1 , rho $=-0.01, p=0.98$; see www. karger.com/doi/10.1159/000513166 for all online suppl. material,). KIM-1/Cr appeared lower in MCD participants compared to other diagnoses (Fig. $2 c, p=0.04$ ).
uKIM-1/Cr also correlated with reversible morphologic changes such as the degree of foot process effacement (Fig. 2d, rho $=0.59, p<0.001$ ) and acute tubular injury (Fig. 2e, rho $=0.31, p=0.04$ ), but not with the degree of chronic changes such as global (online suppl. Fig. 1b, rho $=-0.23, p=0.26$ ) or segmental glomerulosclerosis (online suppl. Fig. $1 c$, rho $=0.28, p=0.17$ ) or TI fibrosis (online suppl. Fig. 1d, rho $=0.06, p=0.72$ ) or atrophy (online suppl. Fig. 1e, rho $=0.04, p=0.81$ ).

Lower baseline uKIM-1/Cr values were associated with more rapid achievement of CR after adjusting for proteinuria, histopathologic diagnosis, and treatment (Fig. 2f, $p=0.01$; Table 2, HR =0.1). For example, adding KIM-1 (high vs. Low, above or below the median) to a multivariable model of time to CR with terms for cohort, UPCR, and treatment was associated with an improvement in Harrell's concordance from 0.74 to 0.82 . Characteristics of high versus low KIM-1 are shown in online suppl. Table 1. A post hoc ROC analysis revealed a uKIM$1 / \mathrm{Cr}$ threshold of $4,364 \mathrm{pg} / \mathrm{mg}$ was the strongest point of discriminating CR versus NR. Coincidentally, this was one value above the median.

\section{Urine POSTN}

Urine POSTN/Cr was associated with higher UPCR (Fig. 3a, rho $=0.60, p<0.001$ ) and UACR (Fig. 3b, rho $=$ $0.56, p<0.001)$. Unlike uKIM-1/Cr, urine POSTN/Cr was associated with lower eGFR (Fig. $3 c$, rho $=0.34, p=0.01$ ). Additionally, urine POSTN/Cr was associated with increased acute tubular injury (Fig. $3 \mathrm{~d}$, rho $=0.49, p=0.001$ ) but was not associated with other morphological features such as global (Fig. 2a, rho $=0.11, p=0.61$ ) or segmental (Fig. $2 b$, rho $=0.31, p=0.13$ ) sclerosis, interstitial fibrosis (Fig. $2 \mathrm{c}$, rho $=0.31, p=0.06$ ), tubular atrophy (Fig. $2 \mathrm{~d}$, rho $=0.29, p=0.06$ ), or foot process effacement (Fig. 2e, rho $=0.28, p=0.11)$. There was no relationship between urine POSTN/Cr and time to CR (Fig. 2f, log-rank, $p=$ 0.17). Although urine POSTN/Cr and uKIM-1/Cr were correlated (Fig. 4, rho $=0.42, p<0.001$ ), uKIM-1/Cr correlated more strongly with outcomes.

\section{TI and Glomerular KIM-1 and POSTN}

In proteinuric individuals, it is possible that the excretion of KIM-1 in the urine might not be indicative of tubular injury per se. The urine biomarker might reflect a combination of marker filtration, impaired tubular marker reabsorption, and/or marker secreted directly from injured tubules. To further assess whether the appearance of KIM-1 in urine reflected intrinsic tubular injury in proteinuric states, we measured the transcript levels of
Wu/Troost/Dai/Nast/Eddy/Wei/Wang/ Gipson/Dell/Gibson/Kretzler/Adler 
Table 2. Time from baseline to CR by KIM-1

\begin{tabular}{|c|c|c|c|}
\hline \multicolumn{4}{|l|}{ Models of continuous KIM-1 (log-2) } \\
\hline Variable & Unadjusted & Full & Reduced \\
\hline KIM-1 (per doubling), HR [95\% CI] & $0.88[0.73-1.05]$ & $0.74[0.51-1.06]$ & $0.76[0.59-0.97]$ \\
\hline \multicolumn{4}{|l|}{ Cohort, HR [95\% CI] } \\
\hline MCD & REF & REF & REF \\
\hline FSGS & $0.02[0.01-0.35]$ & $0.02[0.01-0.14]$ & $0.01[0.01-0.11]$ \\
\hline $\mathrm{MN}$ & $0.04[0.01-0.28]$ & $0.12[0.01-1.30]$ & $0.08[0.01-0.56]$ \\
\hline Other glomerulopathy & $0.04[0.01-0.35]$ & $0.07[0.01-0.81]$ & $0.05[0.01-0.43]$ \\
\hline \multicolumn{4}{|l|}{ Baseline UPCR, HR [95\% CI] } \\
\hline$<1 \mathrm{~g} / \mathrm{g}$ & REF & REF & - \\
\hline $1-3 \mathrm{~g} / \mathrm{g}$ & $0.52[0.13-2.08]$ & $0.43[0.08-2.42]$ & - \\
\hline$>3 \mathrm{~g} / \mathrm{g}$ & $0.41[0.14-1.25]$ & $0.58[0.06-5.72]$ & - \\
\hline \multicolumn{4}{|l|}{ Treated with steroids, HR [95\% CI] } \\
\hline Treated & $0.73[0.25-2.11]$ & $0.96[0.28-3.33]$ & - \\
\hline Untreated & $\mathrm{REF}$ & $\mathrm{REF}$ & - \\
\hline \multicolumn{4}{|l|}{ Harrell's concordance } \\
\hline With KIM-1 & - & 0.79 & 0.76 \\
\hline Without KIM-1 & - & 0.74 & 0.67 \\
\hline \multicolumn{4}{|l|}{$-2 \log$ likelihood } \\
\hline With KIM-1 & - & 82.463 & 83.691 \\
\hline Without KIM-1 & - & 85.290 & 88.258 \\
\hline$p$ value & - & 0.09 & 0.03 \\
\hline \multicolumn{4}{|c|}{ Models of categorical KIM-1 (median split) } \\
\hline Variable & Unadjusted & Full & Reduced \\
\hline \multicolumn{4}{|l|}{ KIM-1, HR [95\% CI] } \\
\hline uKIM-1/UCR >4,215 (pg/mg) & $0.27[0.09-0.79]$ & $0.10[0.02-0.63]$ & $0.13[0.03-0.55]$ \\
\hline uKIM-1/UCR $\leq 4,215$ (pg/mg) & REF & REF & $\mathrm{REF}$ \\
\hline \multicolumn{4}{|l|}{ Cohort, HR [95\% CI] } \\
\hline $\mathrm{MCD}$ & REF & REF & REF \\
\hline FSGS & $0.02[0.01-0.35]$ & $0.02[0.01-0.16]$ & $0.02[0.01-0.14]$ \\
\hline $\mathrm{MN}$ & $0.04[0.01-0.28]$ & $0.15[0.01-1.54]$ & $0.12[0.02-0.82]$ \\
\hline Other glomerulopathy & $0.04[0.01-0.35]$ & $0.06[0.01-0.66]$ & $0.05[0.01-0.46]$ \\
\hline \multicolumn{4}{|l|}{ Baseline UPCR, HR [95\% CI] } \\
\hline$<1 \mathrm{~g} / \mathrm{g}$ & REF & REF & - \\
\hline $1-3 \mathrm{~g} / \mathrm{g}$ & $0.52[0.13-2.08]$ & $0.66[0.13-3.25]$ & - \\
\hline$>3 \mathrm{~g} / \mathrm{g}$ & $0.41[0.14-1.25]$ & $0.72[0.10-5.28]$ & - \\
\hline \multicolumn{4}{|l|}{ Treated with steroids, HR [95\% CI] } \\
\hline Treated & $0.73[0.25-2.11]$ & $1.81[0.44-7.53]$ & - \\
\hline Untreated & $\mathrm{REF}$ & REF & - \\
\hline \multicolumn{4}{|l|}{ Harrell's concordance } \\
\hline With KIM-1 & - & 0.82 & 0.82 \\
\hline Without KIM-1 & - & 0.74 & 0.67 \\
\hline \multicolumn{4}{|l|}{$-2 \log$ likelihood } \\
\hline With KIM-1 & - & 78.413 & 79.248 \\
\hline Without KIM-1 & - & 85.290 & 88.258 \\
\hline$p$ value & - & 0.009 & 0.003 \\
\hline
\end{tabular}

Seven patients were excluded who were in complete remission at baseline ( $n=43 ; n=16$ events). Significant relationship with higher KIM-1 and decreased rate of remission after adjusting for cohort, baseline proteinuria, and steroid therapy during follow-up. CR, complete remission; KIM-1, kidney injury molecule; UPCR, urine protein:Cr ratio; uKIM-1/UCR, urine KIM-1/urine Cr concentration ratio; MCD, minimal change disease; $\mathrm{MN}$, membranous nephropathy; FSGS, focal segmental glomerulosclerosis. 

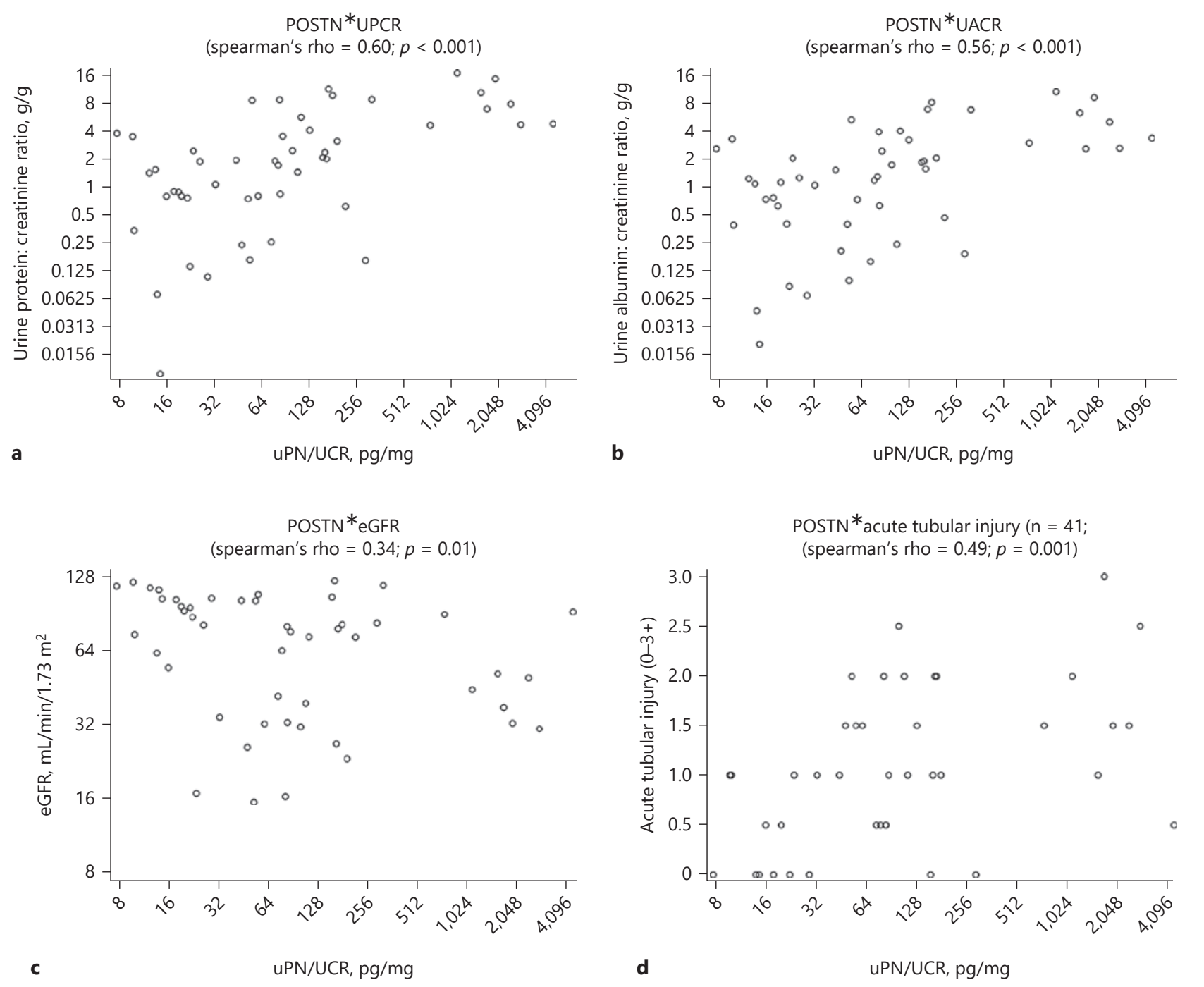

Fig. 3. Baseline urine POSTN correlated with proteinuria, albuminuria, eGFR, and acute tubular injury. a POSTN*UPCR (Spearman's rho $=0.60 ; p<0.001$ ). b POSTN*UACR (Spearman's rho $=0.56 ; p<0.001$ ). c POSTN*eGFR (Spearman's rho $=-0.34 ; p=0.01)$. d POSTN*acute tubular injury $(n=41$; Spearman's rho $=$ $0.49 ; p=0.001$ ). POSTN, periostin; UACR, urine albumin:Cr ratio; UPCR, urine protein:Cr ratio; uPN/UCR, urine periostin/urine $\mathrm{Cr}$ concentration; eGFR, estimated glomerular filtration rate.

KIM-1 in the glomerular and TI compartments from the broader NEPTUNE cohort.

TI KIM-1 transcript levels were associated with more interstitial fibrosis (Fig. 5a, rho $=0.40, p<0.001$ ) and tubular atrophy (Fig. 5b, rho $=0.40, p<0.001$ ). Higher KIM-1TI expression was associated with a modestly higher degree of global sclerosis (Fig. $5 c$, rho $=0.25, p=0.04$ ). However, while glomerular KIM-1 was not associated with interstitial fibrosis (online suppl. Fig. 3a, rho $=0.19, p=0.06$ ), tubular atrophy (online suppl. Fig. 3 b, rho $=0.18, p=0.08$ ), or glob- al sclerosis (online suppl. Fig. $3 c$ rho $=-0.01 p=0.95$ ), it was associated with time to CR (Table 3 ). Higher glomerular KIM-1 expression was associated with longer time to $\mathrm{CR}$, consistent with the uKIM-1 results above.

POSTN expression was not associated with biopsy features, diagnosis, or outcomes (online suppl. Fig. 4). TI POSTN expression was modestly correlated with UACR (rho $=0.24, p=0.02)$. TI and glomerular KIM-1 and glomerular POSTN were associated with UACR (online suppl. Fig. 5). 


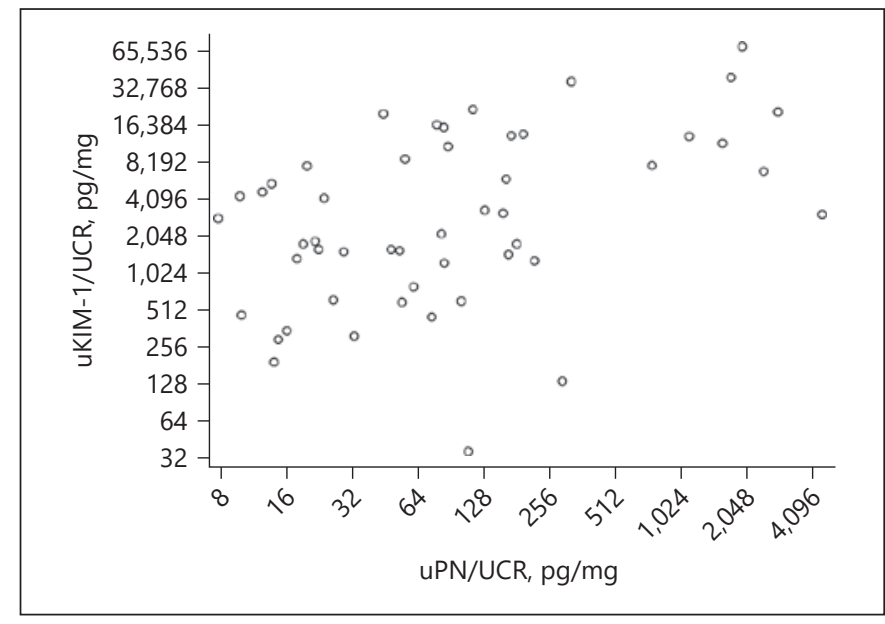

Fig. 4. Urine KIM-1 and POSTN moderately correlated $(p<0.001$; Spearman's rho $=0.42)$. KIM-1, kidney injury molecule; POSTN, periostin; uKIM-1/UCR, urine KIM-1/urine $\mathrm{Cr}$ concentration; $\mathrm{uPN} / \mathrm{UCR}$, urine periostin/urine $\mathrm{Cr}$ concentration.

Correlations among KIM-1, POSTN, and MCP-1 expression are shown in online suppl. Table 2. There were significant correlations between glomerular and TI KIM$1($ rho $=0.53)$, POSTN (rho $=0.23)$, and MCP-1 (rho = 0.33 ). Correlations among these 3 markers were higher and more consistent in the TI than glomerular compartments. For example, higher glomerular POSTN was associated with higher glomerular MCP-1 (rho $=0.19)$ but lower glomerular KIM-1 (rho $=-0.22)$. Higher TI KIM-1 was associated with higher TI POSTN $($ rho $=0.27)$ and higher TI MCP-1 (rho = 0.67). Additionally, TI POSTN was also associated with higher TI MCP-1 (rho $=0.43)$.

\section{Discussion/Conclusion}

Predictive biomarkers are sought as additional determinants to aid clinicians as they prescribe immunosuppressive agents in patients with glomerular disease. Morphological TI changes such as interstitial fibrosis and tubular atrophy have been suggested as better predictors of functional outcome than glomerular changes, even in primary glomerular diseases $[19,20]$. We tested whether KIM-1, a proximal tubule injury marker, or POSTN, a distal tubule injury marker, might serve as candidate biomarkers for this purpose. High baseline uKIM-1/Cr levels correlated with reversible changes such as acute tubular injury and the degree of foot process effacement but not with chronic changes such as the degree of global and segmental sclerosis or TI fibrosis or atrophy. In univariate analyses, high urinary KIM-1/Cr and low TI KIM-1 transcript levels were associated with reversibility of proteinuria in follow-up. After adjusting for baseline proteinuria, histopathologic diagnosis, and treatment, uKIM-1/Cr added value for the prediction of time to CR of proteinuria. Also, KIM-1 was significant in this analysis while baseline proteinuria was not. Our median split analyses found a stronger relationship than those for continuous KIM-1 suggesting a threshold relationship were deleterious effects of KIM-1 are only seen at higher levels. Findings for POSTN were of lesser statistical strength. The data suggest low uKIM-1/Cr protein excretion and low glomerular (KIM-1) transcript levels were associated with the presence of functional and structural changes that were more likely to be modifiable in follow-up related to increased likehood of CR. High TI KIM-1 transcript levels were observed in patients with renal morphological changes of fibrosis, suggesting a relationship between the expression of the tubular injury marker and transition to CKD. Taken together, the data suggest that the combination of high uKIM-1/Cr excretion and low glomerular KIM-1 transcript levels represent the presence of a window during which the acute beneficial effects of KIM-1 on renal recovery may be present but not yet the chronic processes associated with irreversible fibrotic change. The identification of glomerular KIM-1 expression is somewhat novel. Glomerular KIM-1 staining was reportedly induced in parietal epithelial cells in Zucker diabetic fatty rats (fa/fa). Losartan reduced the glomerular KIM-1 protein staining [21]. Our data showing an association between low glomerular KIM-1 transcript levels and renal recovery are consistent with these experimental findings.

Although this study does not provide experimental proof for this interpretation, published data concerning the effects of renal KIM-1 do permit some speculation about mechanism. KIM- 1 is a type 1 transmembrane protein expressed at negligible levels in normal kidney tissue but massively induced in proximal tubule epithelial cells in proteinuric, toxic, and ischemic kidney diseases $[5,22$, 23]. Initial studies demonstrated a role for KIM-1 in mediating a phagocytic phenotype in proximal tubule cells, a function that facilitated the clearance of necrotic and apoptotic tubule cells and intraluminal debris. Thus, transient KIM-1 expression after AKI was posited to facilitate recovery through tissue remodeling after injury [5]. Later experiments demonstrated that in mice, chronic conditional KIM-1 overexpression, even in the absence of kidney injury, caused progressive TI inflammation and fibrosis, mediated at least in part by MCP- 1 . The chronic 


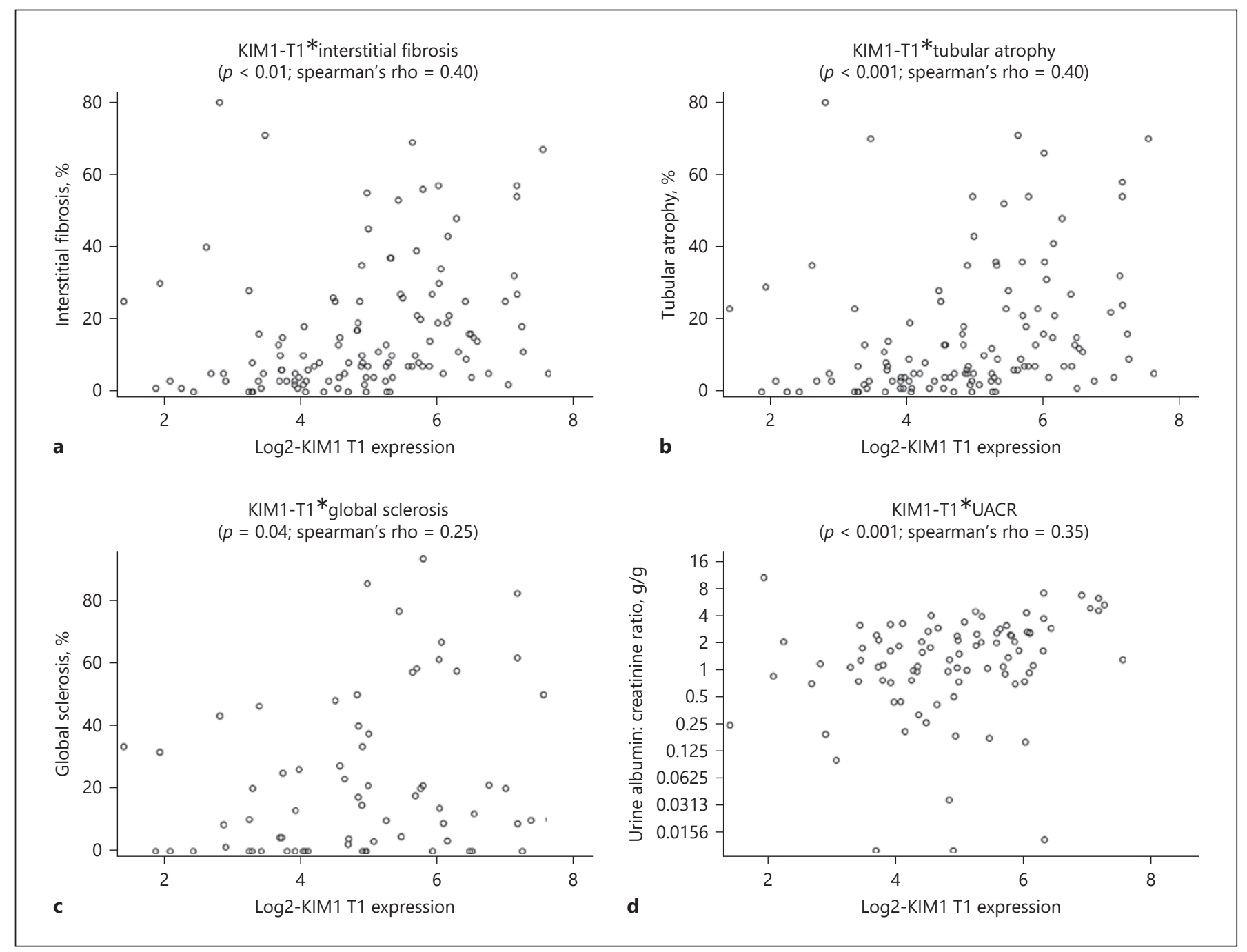

Fig. 5. TI expression KIM-1 correlates with interstitial fibrosis, tubular atrophy, global sclerosis, and albuminuria. a KIM-1-TI*interstitial fibrosis $(p<0.01$; Spearman's rho $=0.40)$. b KIM-1-TI*tubular atrophy $(p<0.001$; Spearman's rho $=0.40)$. c KIM-1-TI*global sclerosis $(p=0.04$; Spearman's rho = 0.25). d KIM-1-TI*UACR $(p<0.001$; Spearman's rho $=0.35)$. KIM-1, kidney injury molecule-1; KIM-1-TI, KIM-1 TI expression; UACR, urine albumin:Cr ratio.

overexpression of KIM-1, which induces the persistence of pro-inflammatory MCP-1, represents an experimentally affirmed mechanism for the transition from adaptive to maladaptive [2]. The proliferation engendered in injured de-differentiated tubular cells to facilitate rapid replication and repair may also facilitate inflammation and sclerosis [24]. Thus, experimental data support the concept that while transient elaboration of KIM-1 may be cytoprotective, chronic KIM-1 expression without resolution leads to TI inflammation and fibrosis [2].

A number of previously published clinical studies examined the role of KIM-1 as a biomarker for renal out- comes with inconsistent results. In the Multi-Ethnic Study of Atherosclerosis (MESA), higher urine KIM-1 excretion (both uncorrected or corrected for urine $\mathrm{Cr}$ ) was associated with more rapid decline in eGFR independent of the degree of albuminuria in a subset of patients with CKD stage 3 and/or rapidly declining renal function. Unlike the more heavily proteinuric patients in the study reported herein, only $6 \%$ of MESA controls and $17 \%$ of subjects with CKD stage 3 had a UACR greater than 30 $\mathrm{mg} / \mathrm{g}$ [25]. In contrast, in the Atherosclerosis Risk in Communities (ARIC) study, urine KIM-1 (uncorrected for $\mathrm{Cr}$ ) correlated with UACR (natural $\log$ ) but was not 
Table 3. Time from baseline to CR by glomerular KIM-1 expression ( $n=111 ; n=60$ events)

\begin{tabular}{|c|c|c|c|}
\hline \multicolumn{4}{|l|}{ Models of continuous KIM-1 (log-2) } \\
\hline Variable & Unadjusted & Full & Reduced \\
\hline KIM-1 (per doubling), HR [95\% CI] & $0.70[0.48-1.02]$ & $0.67[0.45-1.01]$ & $0.65[0.44-0.97]$ \\
\hline \multicolumn{4}{|l|}{ Cohort, HR [95\% CI] } \\
\hline MCD & REF & REF & REF \\
\hline FSGS & $0.17[0.05-0.55]$ & $0.14[0.04-0.45]$ & $0.15[0.05-0.47]$ \\
\hline $\mathrm{MN}$ & $0.25[0.08-0.75]$ & $0.19[0.06-0.61]$ & $0.21[0.07-0.64]$ \\
\hline Other glomerulopathy & $0.20[0.06-0.64]$ & $0.14[0.04-0.47]$ & $0.15[0.05-0.50]$ \\
\hline \multicolumn{4}{|l|}{ Baseline UPCR, HR [95\% CI] } \\
\hline$<1 \mathrm{~g} / \mathrm{g}$ & REF & REF & - \\
\hline $1-3 \mathrm{~g} / \mathrm{g}$ & $1.72[0.72-4.09]$ & $1.83[0.75-4.47$ & - \\
\hline$>3 \mathrm{~g} / \mathrm{g}$ & $0.87[0.42-1.79]$ & $0.91[0.41-2.00]$ & - \\
\hline \multicolumn{4}{|l|}{ Treated with steroids, HR [95\% CI] } \\
\hline Treated & $0.92[0.55-1.55]$ & $1.08[0.62-1.89]$ & - \\
\hline Untreated & $\mathrm{REF}$ & REF & - \\
\hline \multicolumn{4}{|l|}{ Harrell's concordance } \\
\hline With KIM-1 & - & 0.60 & 0.58 \\
\hline Without KIM-1 & - & 0.57 & 0.55 \\
\hline \multicolumn{4}{|l|}{$-2 \log$ likelihood } \\
\hline With KIM-1 & - & 468.219 & 469.858 \\
\hline Without KIM-1 & - & 473.122 & 475.560 \\
\hline$p$ value & - & 0.03 & 0.02 \\
\hline \multicolumn{4}{|c|}{ Models of categorical KIM-1 (median split) } \\
\hline Variable & Unadjusted & Full & Reduced \\
\hline \multicolumn{4}{|l|}{ KIM-1, HR [95\% CI] } \\
\hline High expression $(>2.10)$ & $0.69[0.41-1.15]$ & $0.65[0.38-1.12]$ & $0.62[0.37-1.05]$ \\
\hline Low expression $(\leq 2.09)$ & REF & REF & REF \\
\hline \multicolumn{4}{|l|}{ Cohort, HR [95\% CI] } \\
\hline Minimal change disease & REF & REF & REF \\
\hline FSGS & $0.17[0.05-0.55]$ & $0.15[0.05-0.47]$ & $0.16[0.05-0.51]$ \\
\hline $\mathrm{MN}$ & $0.25[0.08-0.75]$ & $0.22[0.07-0.67]$ & $0.24[0.08-0.73]$ \\
\hline Other glomerulopathy & $0.20[0.06-0.64]$ & $0.15[0.05-0.49]$ & $0.17[0.05-0.55]$ \\
\hline \multicolumn{4}{|l|}{ Baseline UPCR, HR [95\% CI] } \\
\hline$<1 \mathrm{~g} / \mathrm{g}$ & REF & REF & \\
\hline $1-3 \mathrm{~g} / \mathrm{g}$ & $1.72[0.72-4.09]$ & $1.78[0.73-4.35]$ & \\
\hline$>3 \mathrm{~g} / \mathrm{g}$ & $0.87[0.42-1.79]$ & $0.81[0.38-1.76]$ & \\
\hline \multicolumn{4}{|l|}{ Treated with steroids, HR [95\% CI] } \\
\hline Treated & $0.92[0.55-1.55]$ & $1.15[0.65-2.03]$ & \\
\hline Untreated & REF & REF & \\
\hline \multicolumn{4}{|l|}{ Harrell's concordance } \\
\hline With KIM-1 & - & 0.58 & 0.58 \\
\hline Without KIM-1 & - & 0.57 & 0.55 \\
\hline \multicolumn{4}{|l|}{$-2 \log$ likelihood } \\
\hline With KIM-1 & - & 470.658 & 472.419 \\
\hline Without KIM-1 & - & 473.122 & 475.560 \\
\hline$p$ value & - & 0.12 & 0.07 \\
\hline
\end{tabular}

Significant relationship with higher KIM-1 and decreased rate of remission after adjusting for cohort, baseline proteinuria, and steroid therapy during follow-up. CR, complete remission; KIM-1, kidney injury molecule-1; UPCR, urine protein:Cr ratio; uKIM-1/UCR, urine KIM-1/urine Cr concentration ratio; MCD, minimal change disease; MN, membranous nephropathy; FSGS, focal segmental glomerulosclerosis. 
correlated with baseline eGFR and did not predict eGFR decline in a group of subjects with CKD stage 2 and albuminuria $\leq 30 \mathrm{mg} / \mathrm{g}$ at baseline [11]. In the FinnDiane study involving 1,573 diabetic patients followed for 6 years, urine KIM-1 levels correlated inversely with eGFR; in a multivariate regression analysis, after adjusting for diabetes duration, $\mathrm{HbAlc}$, and albumin excretion rate, prognostication was not improved [26]. In patients with various glomerular diseases, KIM-1 staining was observed on the luminal side of de-differentiated proximal tubule cells except in minimal change [23]. KIM-1 staining correlated positively with renal damage indices including inflammation and fibrosis. uKIM-1 correlated negatively with renal function but not with proteinuria [23]. Patients with Henoch-Schonlein purpura (HSP) nephritis $(n=35)$ had higher levels of serum, urine, and immunohistochemically stained renal KIM-1 than patients with HSP without nephritis $(n=20)$. In these HSP patients, serum and urine KIM-1 correlated with BUN and proteinuria and inversely with eGFR at baseline and after 2 years of follow-up. uKIM-1 correlated with indices for TI inflammation, chronic TI change, and crescents. uKIM-1/Cr predicted decline of renal function in followup better than UACR [27]. In 69 nephrotic patients with MN and normal eGFR followed for a median of 39 months, the urine KIM-1 excretion rate was a good predictor of renal functional outcome and proteinuria remission, but it was not more accurate than other tubular markers including NGAL, a1-macroglobulin, or $\beta_{2^{-}}$ macroglobulin [28]. Taken together, few studies have addressed the prognostic value of KIM-1 excretion and/or expression on the outcome of proteinuric renal diseases. Our findings are consistent with many, demonstrating correlations between uKIM-1/Cr, UACR, and UPCR. Our study also corroborated the finding in a cohort of patients with $\mathrm{MN}$ that high uKIM-1/Cr excretion was a good predictor of proteinuria remission [28].

However, in our study, KIM-1 expression levels and urine levels did not correlate, and our study appears to be unique in identifying the combination of high urinary KIM-1/Cr and low glomerular KIM-1 expression as markers of proteinuria remission. One may speculate on the causes of discordance between the KIM-1 tissue expression levels and the urinary excretion. Our study was cross-sectional, not longitudinal. uKIM-1 is associated with renal recovery both in clinical and preclinical studies. In the experimental setting, KIM-1 persistence is associated with progressive injury, inflammation, and sclerosis [2]. The discordance observed between tissue KIM-1 expression and uKIM-1/Cr appearance may have arisen if an increment in tissue KIM-1 expression preceded the biopsy, with tissue recovery underway at the time of the biopsy. This hypothesis could only be tested on serial renal biopsies and urine samples, making it impractical as a clinical study but testable in animal models. In addition, the source of the urine KIM-1 is unknown and may reflect filtration, secretion, and/or local cell elaboration. Thus, it is not surprising that tissue expression may not necessarily correlate well with urine excretion in a cross-sectional observational study.

POSTN is a $90 \mathrm{kD}$ matricellular glycoprotein with roles in wound healing, fibrosis, angiogenesis, cell-matrix adhesion, myocardial healing and fibrosis after infarction, and cancer metastases. It was originally identified in osteoblasts and is induced during nephrogenesis. It is not generally observed in normal adult kidney, but is upregulated in proteinuric, toxic, ischemic, and cystic kidney diseases [3, 29-31]. Few studies report POSTN's role in CKD or as a predictive biomarker [3,31-34]. Our results are consistent with much of the published literature. Overall, in this study, urine POSTN excretion and renal tissue POSTN transcript levels did not improve on the prediction of significant clinical outcomes obtained from routine clinical parameters. Its biological roles in mediating injury and/or repair may still be of interest.

This study has a number of limitations. The crosssectional nature of the study may underlie the apparent discordance between urine KIM-1 excretion and tissue expression. This study also does not resolve the mechanism whereby the combination of high urine KIM-1 excretion coupled with low TI and/or glomerular transcript levels might associate with proteinuria reversibility. We also cannot rule out the possibility that the low signal of KIM-1 expression from the glomerular samples might be from tubular contamination of the samples. This study did not resolve the source of the KIM-1 that is excreted in the urine. Sample size was insufficient to perform extensive multivariable modeling, particularly in the time to CR analyses of KIM-1 (limited by a sample size of 43 participants and 16 events). However, the data are sufficiently strong to warrant further examination at both experimental and clinical levels. Studies of particular interest would address how the persistence of these tubular injury biomarkers contribute to chronic morphologic injury and functional decline. Additional studies may also further address the potential role of urine KIM-1/Cr as a biomarker, as this study had limited power for some end points, especially eGFR, due at least in part to small sample size. We did not stain for KIM-1 in the tissues of these patients with glomerular disease. However, the distribution of KIM-1 staining in both ex-
56

Glomerular Dis 2021;1:45-59 DOI: $10.1159 / 000513166$
Wu/Troost/Dai/Nast/Eddy/Wei/Wang/ Gipson/Dell/Gibson/Kretzler/Adler 
perimental and clinical renal diseases have been established sufficiently to obviate the need to stain for it herein. Another limitation to consider is the generalizability to children with MCD since those with immunosuppression in the weeks before the biopsy were excluded, and most children suspected with MCD are treated prior to biopsy.

We found that high urine excretion and low glomerular transcript levels of KIM-1 appear to identify a subset of patients with glomerular disease who were more likely to enter remission shortly. Conversely, high TI transcript levels correlated with increased TI morphological chronicity. The urinary excretion of KIM-1 may have an impact on the renal microenvironment that facilitates or reflects a capacity for renal recovery; the persistence of KIM-1 expression associates with chronicity of kidney injury. Additional studies are needed to determine whether these phenomena are causative or associational.

\section{Acknowledgements}

We are indebted to the patients and families who generously participated in this research study. We would also like to thank all members of the NEPTUNE consortium listed below: members of the Nephrotic Syndrome Study Network (NEPTUNE). NEPTUNE Enrolling Centers: Cleveland Clinic, Cleveland, OH: J. Sedor*, K. Dell*, M. Schachere", and J. Negrey". Children's Hospital, Los Angeles, CA: K. Lemley* and E. Lim; Children's Mercy Hospital, Kansas City, MO: T. Srivastava* and A. Garrett". Cohen Children's Hospital, New Hyde Park, NY: C. Sethna* and O. Bullaro\#. Columbia University, New York, NY: P. Canetta* and A. Pradhan" . Duke University, Durham, NC: L. Barisoni*; Emory University, Atlanta, GA: L. Greenbaum*, C. Wang**, and C. Kang*. Harbor-University of California Los Angeles Medical Center: S. Adler*, C. Nast**, J. LaPage*. John H. Stroger Jr. Hospital of Cook County, Chicago, IL: A. Athavale* and M. Itteera. Johns Hopkins Medicine, Baltimore, MD: M. Atkinson * and T. Dell ${ }^{*}$. Mayo Clinic, Rochester, MN: F. Fervenza*, M. Hogan**, J. Lieske*, and V. Chernitskiy" Montefiore Medical Center, Bronx, NY: F. Kaskel*, M. Ross*, and P. Flynn". NIDDK Intramural, Bethesda MD: J. Kopp* and J.

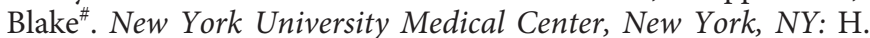
Trachtman*, O. Zhdanova**, F. Modersitzki ${ }^{*}$, and S. Vento ${ }^{\#}$. Stanford University, Stanford, CA: R. Lafayette* and K. Mehta ${ }^{*}$. Temple University, Philadelphia, PA: C. Gadegbeku*, D. Johnstone**, and S. Quinn-Boyle ${ }^{\#}$. University Health Network Toronto: M. Hladunewich**, H. Reich**, P. Ling $^{*}$, and M. Romano ${ }^{*}$. University of Miami, Miami, FL: A. Fornoni* and C. Bidot ${ }^{\#}$; University of Michigan, Ann Arbor, MI: M. Kretzler*, D. Gipson*, A. Williams*, and C. Klida\#. University of North Carolina, Chapel Hill, NC: V. Derebail*, K. Gibson*, E. Cole ${ }^{\#}$, and J. Ormond-Foster ${ }^{\#}$. University of Pennsylvania, Philadelphia, PA: L. Holzman*, K. Meyers**, K. Kallem", and A. Swenson" ${ }^{\#}$. University of Texas Southwestern, Dallas, TX: K. Sambandam*, Z. Wang ${ }^{\#}$, and M. Rogers ${ }^{\#}$. University of Washing- ton, Seattle, WA: A. Jefferson*, S. Hingorani**, K. Tuttle**§, M. Bray \#, E. Pao ${ }^{\#}$, and A. Cooper ${ }^{\# \S}$. Wake Forest University Baptist Health, Winston-Salem, NC: J.J. Lin*, S. Baker. Data Analysis and Coordinating Center: M. Kretzler*, L. Barisoni**, J. Bixler, H. Desmond, S. Eddy, D. Fermin, C. Gadegbeku**, B. Gillespie**, D. Gip-

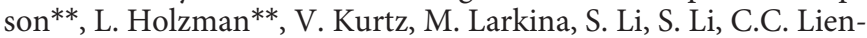
czewski, J. Liu, T. Mainieri, L. Mariani**, M. Sampson**, J. Sedor**, A. Smith, A. Williams, J. Zee. Digital Pathology Committee: Carmen Avila-Casado (University Health Network, Toronto), Serena Bagnasco (Johns Hopkins University), Joseph Gaut (Washington University in St Louis), Stephen Hewitt (National Cancer Institute), Jeff Hodgin (University of Michigan), Kevin Lemley (Children's Hospital of Los Angeles), Laura Mariani (University of Michigan), Matthew Palmer (University of Pennsylvania), Avi Rosenberg (Johns Hopkins University), Virginie Royal (University of Montreal), David Thomas (University of Miami), Jarcy Zee (University of Pennsylvania) Co-Chairs: Laura Barisoni (Duke University) and Cynthia Nast (Cedar Sinai). *Principal investigator; ${ }^{* *}$ co-investigator; and ${ }^{*}$ study coordinator. ${ }^{*}$ Cedars-Sinai Medical Center, Los Angeles, CA. \$Providence Medical Research Center, Spokane, WA.

\section{Statement of Ethics}

This research was carried out according to the Code of Ethics of the World Medical Association (Declaration of Helsinki), and study protocol was reviewed and approved by the University of Michigan Institutional Review Board (HUM00158219). Samples obtained for this study were de-identified and obtained from the central NEPTUNE repository.

\section{Conflict of Interest Statement}

J.T. and D.G. have research funding through the University of Michigan with Complexa Inc., Retrophin Inc., and Goldfinch Bio (D.G. additionally has funding through the University of Michigan with Bristol-Meyers Squibb; J.T. through the University of Michigan with Vertex Pharmaceuticals and Pfizer Inc.).

\section{Funding Sources}

The Nephrotic Syndrome Study Network Consortium (NEPTUNE), U54DK-083912, is a part of the National Institutes of Health (NIH) Rare Disease Clinical Research Network (RDCRN), supported through a collaboration between the Office of Rare Diseases Research, National Center for Advancing Translational Sciences and the National Institute of Diabetes, Digestive, and Kidney Diseases. Additional funding and/or programmatic support for this project has also been provided by NIH funding to the Applied Systems Biology Core at the University of Michigan George M. O'Brien Kidney Translational Core Center (P30 DK081943), the University of Michigan, the NephCure Kidney International and the Halpin Foundation, the Clinical and Translational Science Institute at the David Geffen School of Medicine at UCLA UL1TR001881, and by a gift celebrating the life and work of Dr. 
Henry Shavelle. Dr Qiaoyan Wu was supported by a grant from the National Natural Science Foundation of China (Grant No. 81660118 ) and the scholarship fund of Guangxi education department of China. Jonathan Troost was supported in part by the National Center for Advancing Translational Sciences (NCATS) for the Michigan Institute for Clinical and Health Research (UL1TR002240).

\section{Author Contributions}

NEPTUNE study design, conduct, enrollment of participants: C.N., D.S.G., K.M.D., K.L.G., M.K., and S.A.; conception of overall research questions and approach for secondary analyses of NEP-
TUNE data: Q.W., J.P.T., T.D., B.W., Y.W., and S.A.; measurement and quantification of urine and tissue data: Q.W., T.D., C.N., S.E., Y.W., and S.A.; statistical analyses: J.P.T., S.E., and B.W.; interpretation of results: all authors. Each author contributed important intellectual content during manuscript drafting or revision and agrees to be personally accountable for the individual's own contributions and to ensure that questions pertaining to the accuracy or integrity of any portion of the work, even one in which the author was not directly involved, are appropriately investigated and resolved, including with documentation in the literature if appropriate.

\section{References}

1 Bonventre JV. Kidney injury molecule-1 (KIM-1): a urinary biomarker and much more. Nephrol Dial Transplant. 2009;24(11): 3265-8.

2 Humphreys BD, Xu F, Sabbisetti V, Grgic I, Movahedi Naini S, Wang N, et al. Chronic epithelial kidney injury molecule- 1 expression causes murine kidney fibrosis. J Clin Invest. 2013;123(9):4023-35.

3 Satirapoj B, Wang Y, Chamberlin MP, Dai T, LaPage J, Phillips L, et al. Periostin: novel tissue and urinary biomarker of progressive renal injury induces a coordinated mesenchymal phenotype in tubular cells. Nephrol Dial Transplant. 2012;27(7):2702-11.

4 Mael-Ainin M, Abed A, Conway SJ, Dussaule JC, Chatziantoniou C. Inhibition of periostin expression protects against the development of renal inflammation and fibrosis. J Am Soc Nephrol. 2014;25(8):1724-36.

5 Ichimura T, Asseldonk EJ, Humphreys BD, Gunaratnam L, Duffield JS, Bonventre JV. Kidney injury molecule- 1 is a phosphatidylserine receptor that confers a phagocytic phenotype on epithelial cells. J Clin Invest. 2008; 118(5):1657-68.

6 Gou R, Chen J, Sheng S, Wang R, Fang Y, Yang Z, et al. KIM-1 mediates high glucoseinduced autophagy and apoptosis in renal tubular epithelial cells. Cell Physiol Biochem. 2016;38(6):2479-88.

7 Yang L, Brooks CR, Xiao S, Sabbisetti V, Yeung MY, Hsiao LL, et al. KIM-1-mediated phagocytosis reduces acute injury to the kidney. J Clin Invest. 2015;125(4):1620-36.

8 Han WK, Bailly V, Abichandani R, Thadhani $\mathrm{R}$, Bonventre JV. Kidney injury molecule-1 (KIM-1): a novel biomarker for human renal proximal tubule injury. Kidney Int. 2002; 62(1):237-44.

9 Ichimura T, Bonventre JV, Bailly V, Wei H, Hession CA, Cate RL, et al. Kidney injury molecule-1 (KIM-1), a putative epithelial cell adhesion molecule containing a novel immunoglobulin domain, is up-regulated in renal cells after injury. J Biol Chem. 1998;273(7): 4135-42.
10 Fontanilla J, Han WK. Kidney injury molecule-1 as an early detection tool for acute kidney injury and other kidney diseases. Expert Opin Med Diagn. 2011;5(2):161-73.

11 Bhavsar NA, Köttgen A, Coresh J, Astor BC. Neutrophil gelatinase-associated lipocalin (NGAL) and kidney injury molecule 1 (KIM1) as predictors of incident CKD stage 3: the atherosclerosis risk in communities (ARIC) study. Am J Kidney Dis. 2012;60(2):233-40.

12 Hsu CY, Xie D, Waikar SS, Bonventre JV, Zhang X, Sabbisetti V, et al. Urine biomarkers of tubular injury do not improve on the clinical model predicting chronic kidney disease progression. Kidney Int. 2017;91(1): 196-203.

13 Gadegbeku CA, Gipson DS, Holzman LB, Ojo AO, Song PX, Barisoni L, et al. Design of the nephrotic syndrome study network (NEPTUNE) to evaluate primary glomerular nephropathy by a multidisciplinary approach. Kidney Int. 2013;83(4):749-56.

14 Hogan MC, Lieske JC, Lienczewski CC, Nesbitt LL, Wickman LT, Heyer CM, et al. Strategy and rationale for urine collection protocols employed in the NEPTUNE study. BMC Nephrol. 2015;16:190.

15 Mariani LH, Martini S, Barisoni L, Canetta PA, Troost JP, Hodgin JB, et al. Interstitial fibrosis scored on whole-slide digital imaging of kidney biopsies is a predictor of outcome in proteinuric glomerulopathies. Nephrol Dial Transplant. 2018;33(2):310-8.

16 Lai JY, Luo J, O'Connor C, Jing X, Nair V, Ju W, et al. MicroRNA-21 in glomerular injury. J Am Soc Nephrol. 2015;26(4):805-16.

17 Schmid H, Boucherot A, Yasuda Y, Henger A, Brunner B, Eichinger F, et al. Modular activation of nuclear factor-kappaB transcriptional programs in human diabetic nephropathy. Diabetes. 2006;55(11):2993-3003.

18 Barisoni L, Nast CC, Jennette JC, Hodgin JB, Herzenberg AM, Lemley KV, et al. Digital pathology evaluation in the multicenter nephrotic syndrome study network (NEPTUNE). Clin J Am Soc Nephrol. 2013;8(8): 1449-59.
19 Nath KA. TI changes as a major determinant in the progression of renal damage. Am J Kidney Dis. 1992;20(1):1-17.

20 Bohle A, Mackensen-Haen S, von Gise H, Grund KE, Wehrmann M, Batz C, et al. The consequences of tubulo-interstitial changes for renal function in glomerulopathies. A morphometric and cytological analysis. Pathol Res Pract. 1990;186(1):135-44.

21 Zhao X, Zhang Y, Li L, Mann D, Imig JD, Emmett N, et al. Glomerular expression of kidney injury molecule- 1 and podocytopenia in diabetic glomerulopathy. Am J Nephrol. 2011; 34(3):268-80.

22 Kramer AB, van Timmeren MM, Schuurs TA, Vaidya VS, Bonventre JV, van Goor H, et al. Reduction of proteinuria in adriamycin-induced nephropathy is associated with reduction of renal kidney injury molecule (KIM-1) over time. Am J Physiol Renal Physiol. 2009; 296(5):F1136-45.

23 van Timmeren MM, van den Heuvel MC, Bailly V, Bakker SJ, van Goor H, Stegeman CA. Tubular kidney injury molecule-1 (KIM1) in human renal disease. J Pathol. 2007; 212(2):209-17.

24 Witzgall R, Brown D, Schwarz C, Bonventre JV. Localization of proliferating cell nuclear antigen, vimentin, $\mathrm{c}$-Fos, and clusterin in the postischemic kidney. Evidence for a heterogenous genetic response among nephron segments, and a large pool of mitotically active and dedifferentiated cells. J Clin Invest. 1994;93(5):2175-88.

25 Peralta CA, Katz R, Bonventre JV, Sabbisetti V, Siscovick D, Sarnak M, et al. Associations of urinary levels of kidney injury molecule 1 (KIM-1) and neutrophil gelatinase-associated lipocalin (NGAL) with kidney function decline in the multi-ethnic study of atherosclerosis (MESA). Am J Kidney Dis. 2012;60(6): 904-11.

26 Panduru NM, Sandholm N, Forsblom C, Saraheimo M, Dahlström EH, Thorn LM, et al. Kidney injury molecule- 1 and the loss of kidney function in diabetic nephropathy: a likely causal link in patients with type 1 diabetes. Diabetes Care. 2015;38(6):1130-7. 
27 Zhang Y, Li A, Wen J, Zhen J, Hao Q, Zhang $\mathrm{Y}$, et al. Kidney injury molecule-1 level is associated with the severity of renal interstitial injury and prognosis in adult Henoch-Schönlein purpura nephritis. Arch Med Res. 2017; 48(5):449-58.

28 Maas RJ, van den Brand JA, Waanders F, Meijer E, Goor van H, Peters HP, et al. Kidney injury molecule- 1 and neutrophil gelatinase-associated lipocalin as prognostic markers in idiopathic membranous nephropathy. Ann Clin Biochem. 2016;53(Pt 1):51-7.
29 Ito T, Suzuki A, Imai E, Horimoto N, Ohnishi T, Daikuhara Y, et al. Tornado extraction: a method to enrich and purify RNA from the nephrogenic zone of the neonatal rat kidney. Kidney Int. 2002;62(3):763-9.

30 Sen K, Lindenmeyer MT, Gaspert A, Eichinger F, Neusser MA, Kretzler M, et al. Periostin is induced in glomerular injury and expressed de novo in interstitial renal fibrosis. Am J Pathol. 2011;179(4):175667.

31 Satirapoj B, Tassanasorn S, Charoenpitakchai $\mathrm{M}$, Supasyndh O. Periostin as a tissue and urinary biomarker of renal injury in type 2 diabetes mellitus. PLoS One. 2015;10(4): e0124055.
32 Satirapoj B, Witoon R, Ruangkanchanasetr P, Wantanasiri $\mathrm{P}$, Charoenpitakchai M, Choovichian P. Urine periostin as a biomarker of renal injury in chronic allograft nephropathy. Transplant Proc. 2014;46(1):135-40.

33 Guerrot D, Dussaule JC, Mael-Ainin M, XuDubois YC, Rondeau E, Chatziantoniou C, et al. Identification of periostin as a critical marker of progression/reversal of hypertensive nephropathy. PLoS One. 2012;7(3): e31974.

34 Wantanasiri P, Satirapoj B, Charoenpitakchai M, Aramwit P. Periostin: a novel tissue biomarker correlates with chronicity index and renal function in lupus nephritis patients. Lupus. 2015;24(8):835-45. 